\title{
Herbal Medicine Containing Licorice May Be Contraindicated for a Patient with an HSD1 1 B2 Mutation
}

\author{
Indra Sari Kusuma Harahap, ${ }^{1}$ Naoko Sasaki, ${ }^{1}$ Gunadi, ${ }^{1}$ Surini Yusoff, ${ }^{1}$ Myeong Jin Lee, ${ }^{1}$ \\ Satoru Morikawa, ${ }^{1}$ Noriyuki Nishimura, ${ }^{1}$ Tomohiro Sasaki, ${ }^{2}$ Seiichiro Usuki, ${ }^{3}$ Midori Hirai, ${ }^{4}$ \\ Mika Ohta, ${ }^{5}$ Yutaka Takaoka, ${ }^{5}$ Takashi Nishimoto, ${ }^{6}$ and Hisahide Nishioo \\ ${ }^{1}$ Department of Community Medicine and Social Healthcare Science, Kobe University Graduate School of Medicine, \\ Kobe 650-0017, Japan \\ ${ }^{2}$ Daikakuji Clinic, Kobe University Graduate School of Medicine, Tottori, Japan \\ ${ }^{3}$ Usuki Clinic, Kobe University Graduate School of Medicine, Kobe, Japan \\ ${ }^{4}$ Department of Hospital Pharmacy, Kobe University Graduate School of Medicine, Japan \\ ${ }^{5}$ Division of Applied Genome Science and Bioinformatics, Kobe University Graduate School of Medicine, Kobe, Japan \\ ${ }^{6}$ Nishimoto Clinic, Nishinomiya, Japan \\ Correspondence should be addressed to Indra Sari Kusuma Harahap, nishio@med.kobe-u.ac.jp \\ Received 18 August 2009; Accepted 9 November 2009
}

Copyright ( $) 2011$ Indra Sari Kusuma Harahap et al. This is an open access article distributed under the Creative Commons Attribution License, which permits unrestricted use, distribution, and reproduction in any medium, provided the original work is properly cited.

\begin{abstract}
Licorice ingestion, as well as mutations in the HSD11B2 gene, inhibits $11 \beta$-hydroxysteroid dehydrogenase type 2 (11ßHSD2) enzyme activity, causing the syndrome of apparent mineral corticoid excess (AME). However, the combined effect of licorice ingestion and an HSD11B2 mutation has never been reported, until now. In this study, we demonstrated that licorice ingestion can produce overt hypertension in an individual without medical history of hypertension who is heterozygous for wild-type and mutant HSD11B2 genes. Our patient was a 51-year-old female with serious hypertension who had been taking herbal medicine containing licorice for more than one year. She was clinically diagnosed as having licorice intoxication, because she did not present with hypertension after ceasing the herbal medicine. Molecular analysis showed that she carried a missense mutation, c.40C $>\mathrm{T}$, in HSD11B2. In conclusion, licorice ingestion is an environmental risk factor for hypertension or AME state in patients with a mutation in HSD11B2. Carrying a mutation in HSD11B2 is, conversely, a genetic risk factor for licorice-induced hypertension or AME state. Herbal medicine containing licorice may, therefore, be contraindicated in patients with an HSD11B2 mutation.
\end{abstract}

\section{Introduction}

Licorice (Glycyrrhizae radix) has a long history of use in herbal medicine or traditional medicine of East Asia. Licorice has been used in combination of other herbal ingredients to harmonize and improve the effects of herbal remedy. Many researchers are now studying the effects of licorice with scientific experiments on animals or cultured cells. Recently, cytoprotective effect of licorice has been reported $[1,2]$.

On the other hand, licorice has been known for decades to cause mineralocorticoid-like adverse effects including hypertension, edema, heart failure or hypokalemia, even though patients have low circulating levels of aldosterone [3]. Recent studies revealed that licorice inhibits the enzyme activity of $11 \beta$-hydroxysteroid dehydrogenase type
$2(11 \beta$ HSD2), resulting in a pathological state similar to mineralocorticoid excess named apparent mineralocorticoid excess state (AME; [4-6]). In licorice-induced hypertension, glycyrrhetinic acid, the active substituent of licorice, inhibits $11 \beta$ HSD2 resulting in AME state [3, 7]. Thus, it appears that licorice intoxication is a reversible pharmacological counterpart to AME syndrome, an inherited form of AME state caused by deficiency of $11 \beta \mathrm{HSD} 2$ enzyme activity [3].

The $11 \beta$ HSD2 enzyme mainly converts cortisol into cortisone $[3,7,8]$. Of these two hormones, only cortisol has affinity to mineralocorticoid receptors, and thus induces mineralocorticoid effects. In several hypertensive syndromes, a decrease in $11 \beta \mathrm{HSD} 2$ activity has been described as a pathogenetic mechanism of increased blood pressure. Mutations in the gene HSD11B2 coding for $11 \beta \mathrm{HSD} 2$ compromise 
$11 \beta \mathrm{HSD} 2$ enzyme activity, resulting in juvenile or late-onset forms of the AME syndrome $[9,10]$.

AME syndrome is an autosomal recessive disorder; homozygous or compound heterozygous mutations in the HSD11B2 gene, encoding 11ßHSD2, have been identified in patients [9-14]. Heterozygosity for wild and mutant HSD11B2 alleles does not cause AME state or hypertension $[9,15]$, although some parents of AME patients are heterozygous for a mutation presented with a phenotype indistinguishable from "essential" hypertension [10].

However, the combined effect of licorice ingestion and the HSD11B2 mutation has never been reported. Herbal medicine is very popular in Japan and many remedies available in Japan contain licorice. However, the frequency of the HSD11B2 mutation carriers in the Japanese population is $\sim 1 \%$ [16]. Thus, it is curious that HSD11B2 mutation has not been reported among Japanese patients with licorice intoxication, and that the combined effect of licorice ingestion and the HSD11B2 mutation has never been previously discussed.

In this study, we demonstrated that licorice ingestion can produce overt hypertension in an individual who is heterozygous for wild-type and mutant HSD11B2 genes with no history of hypertension. This indicates a combined effect of licorice ingestion and the HSD11B2 mutation.

\section{Methods}

2.1. Patient. The patient was a 51-year-old female with a two-year history of postmenopausal syndrome. Her weight and height were $49 \mathrm{~kg}$ and $153 \mathrm{~cm}$. To improve the symptoms of postmenopausal syndrome, she had been taking an herbal medicine, Shimotu-ka-ninjin-kanzo-to, that is, four herbs (Rehmannia, Cnidium, Paeonia and Angelica) plus ginseng and licorice decoction for more than one year. Her daily ingestion of licorice was less than $1.0 \mathrm{~g}$. She did not show hypertension in her medical history. Her systolic blood pressure was usually measured to be less than $130 \mathrm{mmHg}$. On April 15, 2006, she was admitted to a medical office with a heavy headache lasting several hours. On examination, her blood pressure while sitting was $190 / 110 \mathrm{mmHg}$. She was just instructed to rest quietly in bed at home and to stop taking the herbal medicine. Her blood pressure stabilized to within normal limits the following day. She did not take the herbal medicine after this episode and did not experience hypertension again. Although other clinical examinations apart from blood pressure measurement were not performed, it was strongly suspected that her paroxysmal hypertension was associated with licorice intoxication.

2.2. Molecular Analysis. Informed consent was obtained from the patient before the analysis. Genomic DNA was extracted from EDTA whole blood sample using a commercially available DNA extraction kit. Each exon of HSD11B2 was PCR-amplified with the primer pairs reported previously [9], followed by denaturing highperformance liquid chromatography (DHPLC) screening and direct sequencing according to our previous reports

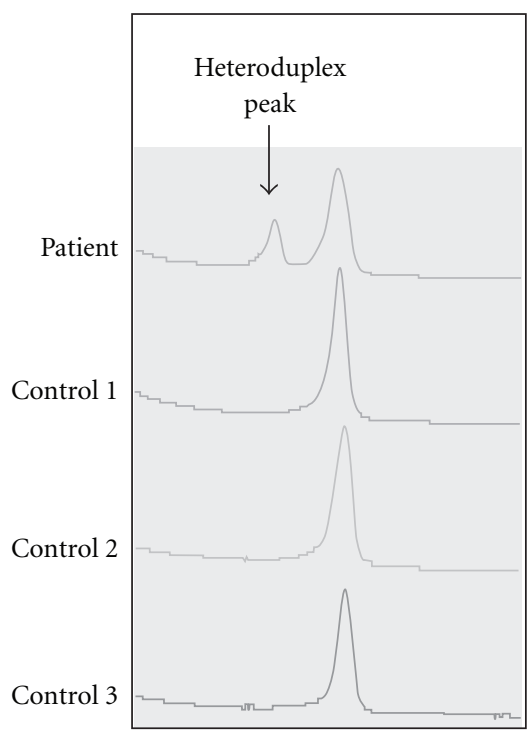

FIGURE 1: DHPLC screening for a mutation in the HSD11B2 gene. Screening showed a small and large peak on the chromatogram of HSD11B2 exon 1, while controls showed a single peak. The small peak indicated heteroduplex DNA formation, suggesting the presence of a mutation in the patient's HSD11B2 exon 1.

[17]. DHPLC screening for the HSD11B2 mutation, c.40C $>$ T, was performed with primers as described below: $\quad 5^{\prime}$-GCTGGGCCGCCATGGAGC-3' and $5^{\prime}$ CGGGACAACGCGATCCAGC-3'.

\section{Results}

3.1. DHPLC Screening for a Mutation in HSD11B2. DHPLC screening showed that the patient had a nucleotide mutation in the PCR fragment including HSD11B2 exon 1. As shown in Figure 1, the patient had two peaks in her DHPLC chart of the PCR fragment including HSD11B2 exon 1. This finding suggested that the patient carried nucleotide changes in this region. However, DHPLC screening did not disclose what kind of mutation was present in the patient.

3.2. Identification of the Mutation in HSD11B2. To identify the mutation in the patient, we conducted a direct DNA sequencing analysis of the PCR fragment including HSD11B2 exon 1. As shown in Figure 2, the patient carried a missense mutation, c.40C $>$ T, leading to an amino acid substitution from leucine to phenylalanine at position 14 of $11 \beta \mathrm{HSD} 2$ (p.L14F). Figure 2 also demonstrates that the patient was heterozygous for normal and mutant alleles. In other regions of the HSD11B2 gene, no nucleotide change was observed in the patient.

\section{Discussion}

4.1. Heterozygous Mutation in HSD11B2. We identified a heterozygous missense mutation, c.40C $>\mathrm{T}$, in the HSD11B2 


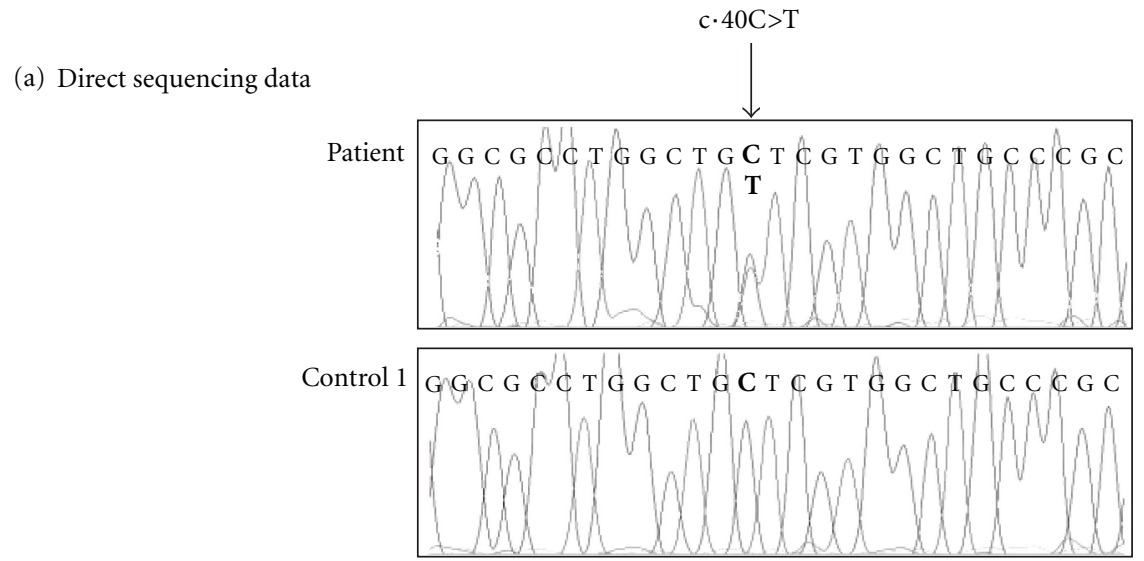

(b) Amino acid sequence surrounding the mutation

\begin{tabular}{|c|c|c|c|c|c|c|c|c|c|c|}
\hline Codon number & & 10 & 11 & 12 & 13 & 14 & 15 & 16 & 17 & 18 \\
\hline \multirow[t]{2}{*}{ Wild-type } & Nucleotides & GGC & GCC & TGG & CTG & $\underline{\text { CTC }}$ & GTG & GCT & GCC & CGC \\
\hline & Amino acids & G & A & $\mathrm{W}$ & $\mathrm{L}$ & $\mathrm{L}$ & $\mathrm{V}$ & A & $\mathrm{A}$ & $\mathrm{R}$ \\
\hline \multirow[t]{2}{*}{ Mutant-type } & Nucleotides & GGC & GCC & TGG & CTG & $\underline{\mathrm{TTC}}$ & GTG & GCT & GCC & CGC \\
\hline & Amino acids & G & A & $\mathrm{W}$ & $\mathrm{L}$ & F & $\mathrm{V}$ & A & A & $\mathrm{R}$ \\
\hline
\end{tabular}

FIGURE 2: Direct DNA sequencing of HSD11B2 exon 1 (a) and amino acid sequence surrounding the mutation (b). DNA sequencing showed heterozygous status for the wild-type ( $\mathrm{C}$ at nucleotide position 40 ) and mutant ( $\mathrm{T}$ at nucleotide position 40 ) sequences.

gene in a Japanese patient with licorice-induced hypertension. Kamide et al. [16] first reported the c.40C $>\mathrm{T}$ in HSD11B2 exon 1 in the Japanese population. They found the mutation in 11 out of 2433 hypertensive individuals and in 8 out of 2175 normotensive individuals. Although there have not been reports of enzyme activity of mutated $11 \beta \mathrm{HSD} 2$ nor AME patients homozygous for the mutation, this mutation occurs in the amino acid residue that is highly conserved among three different species (human, mouse and rabbit), indicating that the mutation may affect $11 \beta$ HSD 2 activity [16].

In general, heterozygosity for wild and mutant HSD11B2 alleles does not play a critical role in the pathogenesis of AME state in most cases [9, 15]. Lavery et al. [10] noted that the mothers of AME patients heterozygous for missense mutations presented with a phenotype indistinguishable from essential hypertension. Taken together, all of these findings suggested that one copy of normal HSD11B2 allele may guarantee enough levels of $11 \beta \mathrm{HSD} 2$ enzyme activity to prevent serious AME syndrome and that factors other than HSD11B2 mutation may be required for the development of hypertension or AME in heterozygous patients.

\subsection{Licorice Ingestion as an Environmental Risk Factor.} A question then arises as to why our patient showed hypertension despite being heterozygous for an HSD11B2 mutation. The most likely explanation is that the licorice in the herbal medicine inhibited the residual enzyme activity of normal $11 \beta$ HSD2 in the patient, causing paroxysmal heavy headache as a symptom of AME state. Thus, we can speculate that licorice ingestion contributed to the development of hypertension in this patient (Figure 3 ).

4.3. Carrying a Mutation in HSD11B2 as a Genetic Risk Factor. It is also noteworthy that our patient had been ingesting no more than $1.0 \mathrm{~g}$ of licorice per day for only one year before the paroxysmal hypertension. Yasue et al. [18] reported that a 93-year-old hypertensive female, taking herbal medicines containing licorice, suffered from severe hypokalemia, rhabdomyolysis, muscle paralysis and respiratory impairment due to a serious AME state. She had been ingesting $5.0 \mathrm{~g}$ of licorice daily for the last seven years. Gerritsen et al. [19] also reported that a 44-year-old previously healthy female, was admitted to the emergency department following resuscitation from ventricular fibrillation. She had been ingesting 250-500 g of licorice daily for the several years. Compared with these patients, our patient suffered from licorice intoxication after consuming a very small amount of licorice, which suggested that the presence of an HSD11B2 mutation contributed to the development of her hypertension (Figure 3).

It may be hazardous for such patients with an HSD11B2 mutation to take herbal medicine containing licorice. To avoid serious side effects of licorice, pharmacogenetic approaches are required. It has been reported that an Ato-G change at nucleotide 1555 in mitochondrion DNA is associated with aminoglycoside-induced hearing loss [20]. Now, genetic testing of mitochondrion DNA is widely used to 


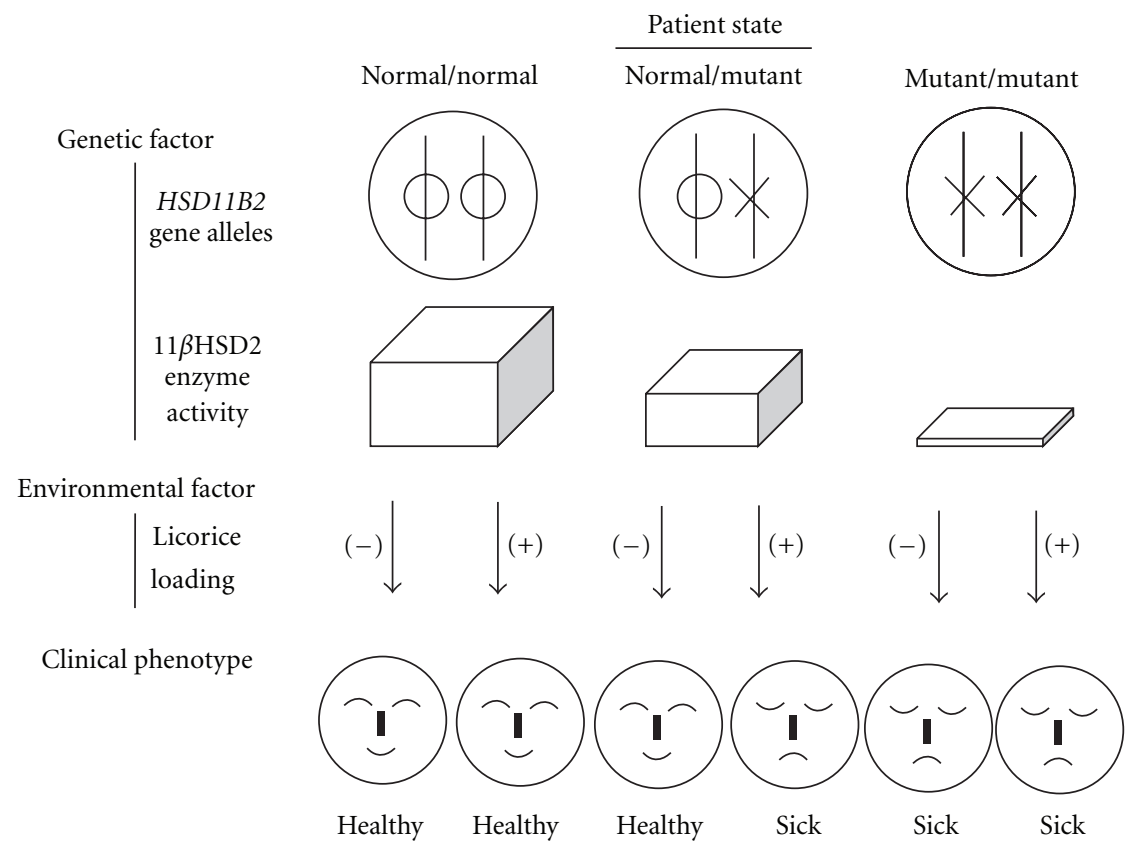

FIGURE 3: Schematic drawing of the pathogenesis of the patient. The patient was heterozygous for a mutation in HSD11B2 ("normal/mutant" alleles in the figure), suggesting that her $11 \beta \mathrm{HSD} 2$ enzyme activity in the kidney was reduced. Without licorice loading, she was able to keep her healthy condition. However, with licorice loading, she showed serious hypertension.

avoid hearing loss caused by aminoglycosides [21]. Similarly, genetic testing of HSD11B2 will be useful to avoid licoriceinduced hypertension or AME state.

\section{Conclusion}

We applied a pharmacogenetic approach to licorice intoxication and were the first to identify the combined effect of licorice ingestion and an HSD11B2 mutation. Licorice ingestion is an environmental risk factor for hypertension or AME state in patients with a mutation in HSD11B2. Carrying a mutation in HSD11B2 is therefore, conversely, a genetic risk factor for licorice-induced hypertension or AME state. Herbal medicine containing licorice may be contraindicated for patients with an HSD11B2 mutation. Genetic testing to detect individuals with an HSD11B2 mutation will be useful to avoid licorice-induced adverse effects.

\section{Acknowledgments}

The authors thank Ms. Chiyo Hayashi and Ms. Reiko Nagao for their technical assistance. This study was supported by a grant-in-Aid for Scientific Research Ministry of Science, Culture and Sports of Japan.

\section{References}

[1] S. C. Kim, S. J. Park, J. R. Lee, J. C. Seo, C. H. Yang, and S. H. Byun, "Cytoprotective activity of Glycyrrhizae radix extract against arsenite-induced cytotoxicity," Evidence-Based Complementary and Alternative Medicine, vol. 5, no. 2, pp. 165-171, 2008.
[2] J. R. Lee, S. J. Park, H.-S. Lee et al., "Hepatoprotective activity of licorice water extract against Cadmium-induced toxicity in rats," Evidence-Based Complementary and Alternative Medicine, vol. 6, no. 2, pp. 195-201, 2009.

[3] P. C. White, T. Mune, and A. K. Agarwal, " $11 \beta$-Hydroxysteroid dehydrogenase and the syndrome of apparent mineralocorticoid excess," Endocrine Reviews, vol. 18, pp. 135-156, 1997.

[4] P. M. Stewart, R. Valentino, A. M. Wallace, D. Burt, C. H. L. Shackleton, and C. R. W. Edwards, "Mineralocorticoid activity of liquorice: 11-beta-hydroxysteroid dehydrogenase deficiency comes of age," The Lancet, vol. 2, no. 8563, pp. 821-824, 1987.

[5] M. A. MacKenzie, W. H. Hoefnagels, R. W. Jansen, T. J. Benraad, and P. W. Kloppenborg, "The influence of glycyrrhetinic acid on plasma cortisol and cortisone in healthy young volunteers," Journal of Clinical Endocrinology \& Metabolism, vol. 70, pp. 1637-1643, 1990.

[6] F. Hammer and P. M. Stewart, "Cortisol metabolism in hypertension," Best Practice and Research: Clinical Endocrinology and Metabolism, vol. 20, no. 3, pp. 337-353, 2006.

[7] P. M. Stewart, J. E. T. Corrie, C. H. L. Shackleton, and C. R. W. Edwards, "Syndrome of apparent mineralocorticoid excess. A defect in the cortisol-cortisone shuttle," The Journal of Clinical Investigation, vol. 82, no. 1, pp. 340-349, 1988.

[8] S. H. M. Van Uum, A. R. M. M. Hermus, P. Smits, T. Thien, and J. W. M. Lenders, "The role of $11 \beta$-hydroxysteroid dehydrogenase in the pathogenesis of hypertension," Cardiovascular Research, vol. 38, no. 1, pp. 16-24, 1998.

[9] T. Mune, F. M. Rogerson, H. Nikkila, A. K. Agarwal, and P. C. White, "Human hypertension caused by mutations in the kidney isozyme of $11 \beta$ - hydroxysteroid dehydrogenase," Nature Genetics, vol. 10, no. 4, pp. 394-399, 1995.

[10] G. G. Lavery, V. Ronconi, N. Draper et al., "Late-onset apparent mineralocorticoid excess caused by novel compound heterozygous mutations in the HSD11B2 gene," Hypertension, vol. 42, no. 2, pp. 123-129, 2003. 
[11] R. C. Wilson, M. D. Harbison, Z. S. Krozowski et al., "Several homozygous mutations in the gene for 11 beta-hydroxysteroid dehydrogenase type 2 in patients with apparent mineralocorticoid excess," Journal of Clinical Endocrinology \& Metabolism, vol. 80, pp. 3145-3150, 1995.

[12] R. C. Wilson, S. Dave-Sharma, J.-Q. Wei et al., "A genetic defect resulting in mild low-renin hypertension," Proceedings of the National Academy of Sciences of the United States of America, vol. 95, no. 17, pp. 10200-10205, 1998.

[13] S. Dave-Sharma, R. C. Wilson, M. D. Harbison et al., "Examination of genotype and phenotype relationships in 14 patients with apparent mineralocorticoid excess," Journal of Clinical Endocrinology \& Metabolism, vol. 83, no. 7, pp. 22442254, 1998.

[14] A. Odermatt, B. Dick, P. Arnold et al., "A mutation in the cofactor-binding domain of 11beta-hydroxysteroid dehydrogenase type 2 associated with mineralocorticoid hypertension," Journal of Clinical Endocrinology \& Metabolism, vol. 86, pp. 1247-1252, 2001.

[15] P. M. Stewart, Z. S. Krozowski, A. Gupta et al., "Hypertension in the syndrome of apparent mineralocorticoid excess due to mutation of the $11 \beta$-hydroxysteroid dehydrogenase type 2 gene," The Lancet, vol. 347, no. 8994, pp. 88-91, 1996.

[16] K. Kamide, Y. Kokubo, H. Hanada et al., "Genetic variations of HSD11B2 in hypertensive patients and in the general population, six rare missense/frameshift mutations," Hypertension Research, vol. 29, no. 4, pp. 243-252, 2006.

[17] Gunadi, K. Miura, M. Ohta et al., "Two novel mutations in the ED1 gene in Japanese families with X-linked hypohidrotic ectodermal dysplasia," Pediatric Research, vol. 65, no. 4, pp. 453-457, 2009.

[18] H. Yasue, T. Itoh, Y. Mizuno, and E. Harada, "Severe hypokalemia, rhabdomyolysis, muscle paralysis, and respiratory impairment in a hypertensive patient taking herbal medicines containing licorice," Internal Medicine, vol. 46, no. 9, pp. 575-578, 2007.

[19] K. G. Gerritsen, J. Meulenbelt, W. Spiering, I. P. Kema, A. Demir, and V. J. van Driel, "An unusual cause of ventricular fibrillation,” The Lancet, vol. 373, no. 9669, p. 1144, 2009.

[20] T. R. Prezant, J. V. Agapian, M. C. Bohlman et al., "Mitochondrial ribosomal RNA mutation associated with both antibiotic- induced and non-syndromic deafness," Nature Genetics, vol. 4, no. 3, pp. 289-294, 1993.

[21] S.-I. Usami, S. Abe, H. Shinkawa, Y. Inoue, and T. Yamaguchi, "Rapid mass screening method and counseling for the $1555 \mathrm{~A} \rightarrow \mathrm{G}$ mitochondrial mutation," Journal of Human Genetics, vol. 44, no. 5, pp. 304-307, 1999. 


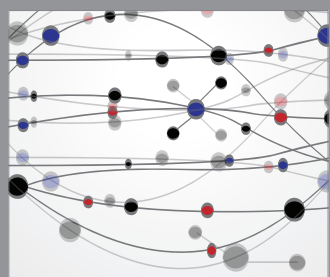

The Scientific World Journal
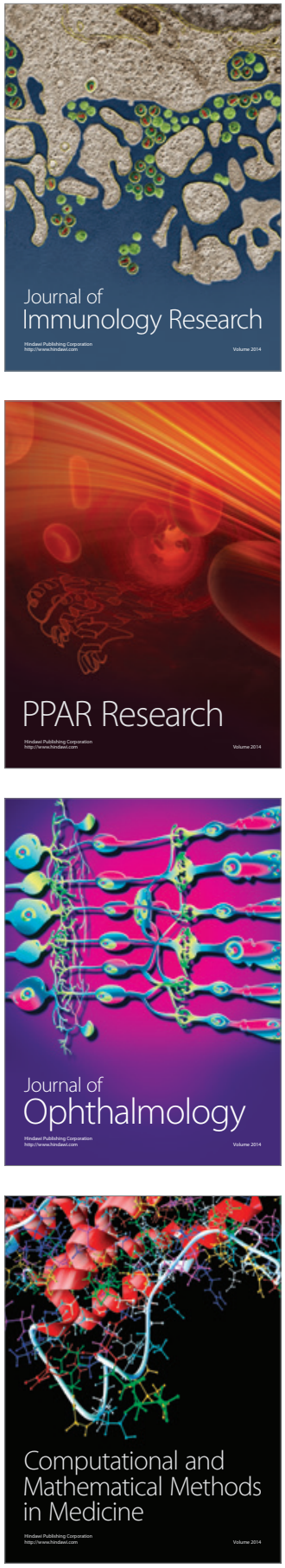

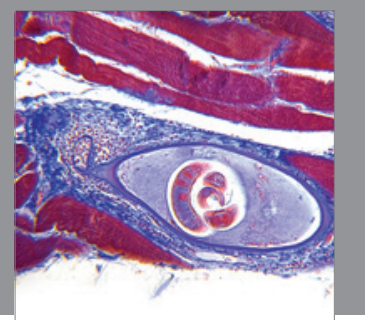

Gastroenterology

Research and Practice
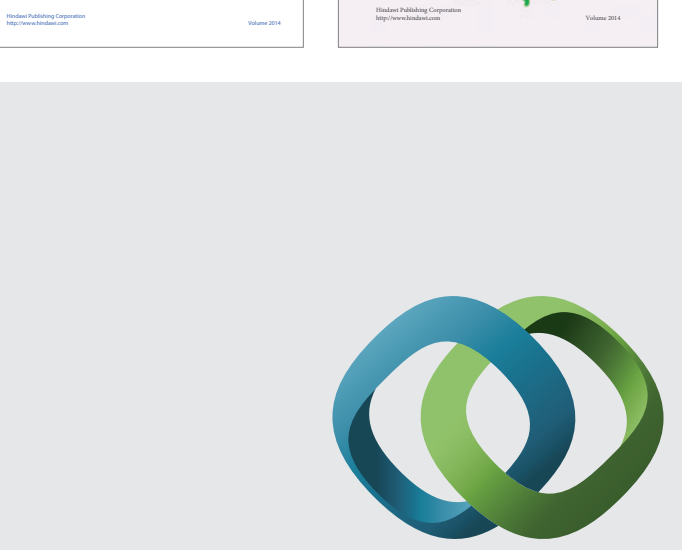

\section{Hindawi}

Submit your manuscripts at

http://www.hindawi.com
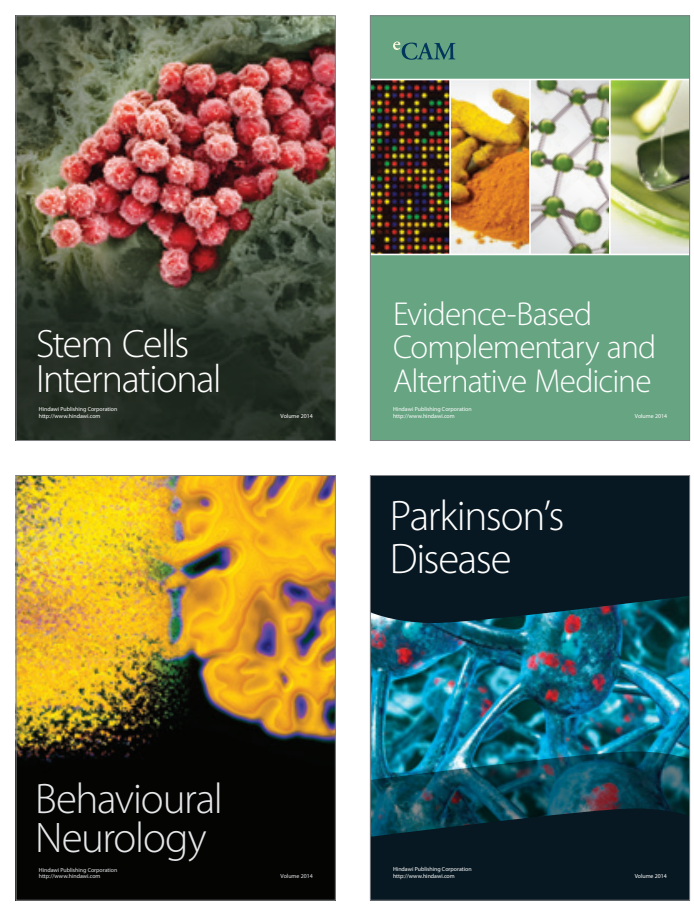

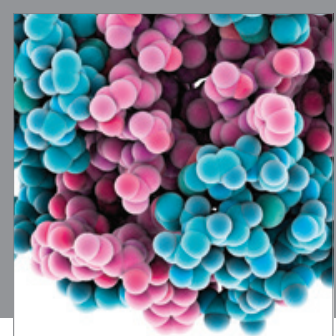

Journal of
Diabetes Research

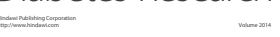

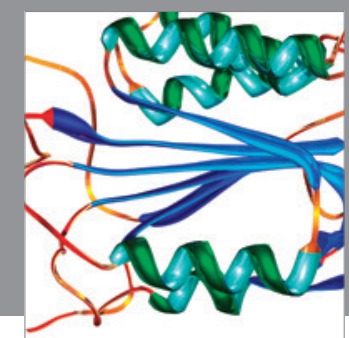

Disease Markers
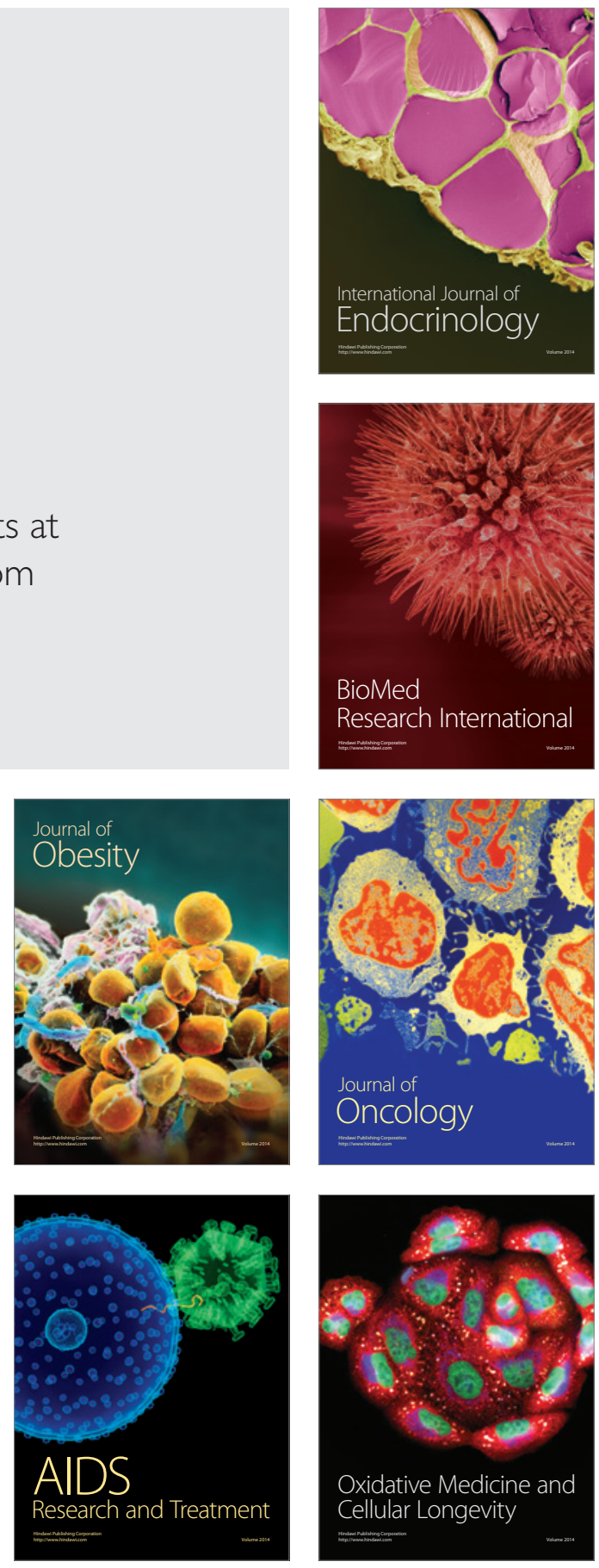